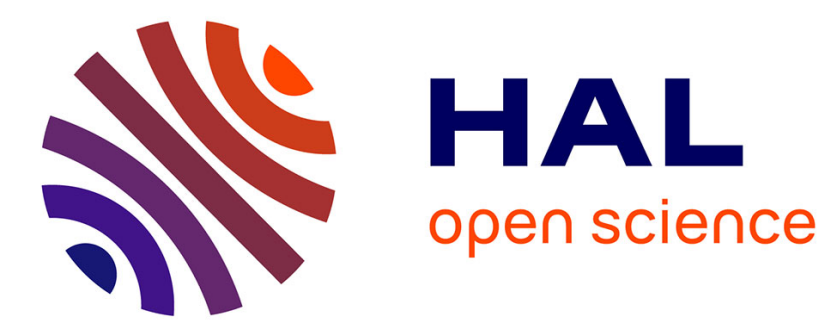

\title{
Self-adaptive modal control for time-varying structures
}

Fengyan Deng, Didier Rémond, Luc Gaudiller

\section{To cite this version:}

Fengyan Deng, Didier Rémond, Luc Gaudiller. Self-adaptive modal control for time-varying structures. Journal of Sound and Vibration, 2011, 330 (14), pp.3301-3315. 10.1016/j.jsv.2011.01.004 . hal00591410

\section{HAL Id: hal-00591410 \\ https://hal.science/hal-00591410}

Submitted on 1 Jun 2019

HAL is a multi-disciplinary open access archive for the deposit and dissemination of scientific research documents, whether they are published or not. The documents may come from teaching and research institutions in France or abroad, or from public or private research centers.
L'archive ouverte pluridisciplinaire HAL, est destinée au dépôt et à la diffusion de documents scientifiques de niveau recherche, publiés ou non, émanant des établissements d'enseignement et de recherche français ou étrangers, des laboratoires publics ou privés. 


\title{
Self-adaptive modal control for time-varying structures
}

\author{
Fengyan DENG, Didier RÉMOND, Luc GAUDILLER* \\ Université de Lyon, CNRS, INSA-Lyon, LaMCoS, UMR5259, F-69621,Villeurbanne, France \\ * Corresponding author: luc.gaudiller@insa-lyon.fr
}

\begin{abstract}
For the past several years, modal controllers are widely studied and used in the field of vibration or vibro-acoustics control. They are efficient but not robust, because these methods involve a reconstructor based on a modal truncation. When the dynamic behavior of the structure change, the controller and reconstructor must be updated to cope with the changes in the structure behavior, in order to maintain both performance and robustness. A solution is adaptive control but this approach needs some specific information not generally available particularly in the case of undergone modifications. This paper deals with a self-adaptive modal control based on a real-time identifier, which avoid the need of specific information. The identifier permits to update the controller and the reconstructor according to the changes of modal characteristics of time-varying structures. A classical algorithm of identification is used to obtain a state space model with an unspecified state vector. Then, based on this model, a well adapted transformation is carried out to get the modal characteristics from the expression of complex modes, including the mode shapes. As a criterion of running identification, the value of "variance-accounted for" (VAF) is employed to carry out the identifier only when the initial or previous model is not enough exact. A Linear Quadratic Gaussian Algorithm is employed in such a way that the control and observer can be optimized according to the updated modal model. By this way, a self-adaptive modal control is completed and can demonstrate some smart properties. The proposed methodology is carried out on a simple but representative time-varying mechanical discrete structure. An inertia modification leads not only to low modal frequency shifts but also to inversion of a mode shape which is shown to lead to unstable configuration when control system is not
\end{abstract}


updated. The overall procedure will be described through simulations and performed for different operating conditions, which will prove that mode shapes have to be precisely determined and updated in the controller and observer to guarantee a robust modal control with high performance in spite of the changes of structure.

Keywords: self-adaptive control / modal control / real-time identification / mode shape change / time-varying structures

\section{Introduction}

In the past decades, active control has been studied and widely applied to many applications in vibration control. In the case of flexible structures, two main types of control strategy emerge. For the first approach, a collocated actuator/sensor control ensures unconditional asymptotic stability [1] when actuator and sensor are ideal. Even if this control is robust, it requires a lot of actuator/sensor pairs to get a high performance control on several modes due to its local actuation area. On the opposite, active modal control is based on a model and recognized for efficiency but it is not ensured to be robust [2]. This global control can target specific modes with a few actuators and sensors [3] and permits to reduce external actuation energy, on-board mass and volume of amplifier [4],[5].

The boundary conditions or structural variation can generate changes of modal characteristics like natural frequencies, modal damping coefficients, but what's more on mode shapes. Therefore, in order to improve the robustness of active control, several methods have been proposed, which can be used in the case of smooth behaviour change.

First of all, robust control can deeply improve robustness of controlled structure [6] but it reduces the overall performances. Robust control is not well adapted to modal formulation which is widely used in the mechanical field. Moreover, for a dynamical behaviour with large modifications like mode shape inversion, robust control can be inefficient and not well adapted. 
Other methods have been developed considering performance and robustness at the same time. Nonlinear control is established and suitable for large modifications and nonlinearities of the dynamical behaviour. In that case, the system can be nonlinear, timevariant, or both of them [7]. This solution is only functional for particular cases of nonlinearities and designed for a given structure. To be efficient, nonlinear control needs some supplementary information to reconstruct the change of structure.

Adaptive control has been extensively studied in the last decades. As nonlinear control, relevant information is needed [8] and should be known a priori or measured. In other methods like Multiple Model Adaptive Control [9], the limits of behaviour change should be known to design a set of controllers which can be defined on the different linearized behaviours of the nonlinear structure. Adaptive modal control offers a trade-off between high performances and robustness [10]. The drawback of this approach is the need of supplementary information concerning the characteristics for update the control. Without this information, all methods in the field of adaptive control will be ineffective.

Recently, the idea of control-oriented identification [11] has been proposed where the model of actual structure is used to design a parameterized controller. Then the error between the measured output and the output of the controlled reference model is minimized to realize a parameter optimization problem. These identified parameters can be used to update the controller, offering constant performances even with actual structure modifications. Consequently, an approach based on an iterative identification and control is developed [12] where an identified model is obtained by using the closed-loop data through online measurements in order to update controller. So the closed-loop identification is developed to reconstruct a model with behaviour changes, like direct, indirect, joint input-output [13] and extended instrumental variable (IV) methods [14]. For indirect approaches, in the case of strongly damped structures thanks to the controller, identification methods will not present enough accuracy. Another approach is Iterative Feedback Tuning (IFT) [15], where an 
optimization is carried out directly on the controller parameters without the identification step altogether. But in the above methods, the controlled system is required to be stable. In such black box approaches, the causes of instability cannot be known due to the lack of modelling. On the contrary, direct identification approaches aims at finding a model of the structure which is lightly damped. Therefore, identification results can be accurate and this corresponding model reconstruction also permits to identify changes which can induce instability for the controlled structure.

A particularly performing model in the case of vibration control is based on modal description which limits the number of sensors and actuators and the model size [3]. Modal characteristics like natural frequencies, modal damping ratio, and especially mode shapes need to be known to design modal controller.

However, modal control can be instable if the model doesn't correspond to actual structure particularly when the structure is changing. As an example, mass or stiffness configuration modifies modal characteristics like natural frequencies and especially mode shapes. In this case, the adaptive modal control can be a solution. These changes need to be known to update the modal controller but need specific information allowing the reconstruction of characteristic change. Recently, Luş and De Angelis [16] provided a unified framework to reconstruct model by some methods considering a full or a non-full set of sensors or actuators. By their approach, the modal characteristics can be deduced based on the complex modes stemming from an identified state space model.

For actual control system, identification should be carried out only when the model is not enough exact. So the value of "Variance-Accounted For" (VAF) [17] is employed to evaluate the difference between the identified model and the actual structure and consecutively to carry out identification process when needed.

Based on the above mentioned principles, self-adaptive modal control for time-varying structures is proposed in this paper. Combining online real-time identification and adaptive 
modal control, this method can be used in order to design an effective and stable controller for time-varying structures.

First of all, the modal description of a structure is presented in section 2 , and then a modal control combining a real-time identification, which can be triggered from a specific criterion, is proposed as self-adaptive modal control. Section 3 is devoted to the description of the proposed method through a simple mechanical structure. The chosen example is a 3 Degrees of Freedom (DoF) mechanical structure in which inertia of the third DoF can be changed with time, inducing frequency shifts and more specifically mode shape inversion. Results of simulation are presented and a conclusion is summarized in the last section.

\section{Self-adaptive modal control}

The proposed self-adaptive modal control deals with the control of structure subjected to variation of its physical characteristics. It is supposed that these changes can not be measured or known a priori and they induce a variation of dynamical behaviour. This dynamical behaviour can be described in a modal form as a simplified model of a complex structure. Instability of such a controlled structure can be induced by modification in eigen frequencies, modal damping ratio and especially mode shapes. Some conventional algorithms of modal control and modal identification used in this paper are recalled and then the principle and realization of self-adaptive modal control are proposed as the kernel of this method.

\subsection{Modal control}

Actual structure can be modelled by $N$ Degrees of Freedom (DoF), so second-order differential equation is presented to describe this structure as:

$$
\mathbf{M} \ddot{\boldsymbol{\delta}}+\Gamma \dot{\boldsymbol{\delta}}+\mathbf{K} \boldsymbol{\delta}=\mathbf{F}
$$

where $\mathbf{M}, \Gamma, \mathbf{K}$ are mass, damping and stiffness matrices, respectively; $\boldsymbol{\delta}$ and $\mathbf{F}$ are the displacement of DoF and the external force vectors, respectively. After the classical modal 
change of variable $\boldsymbol{\delta}=\boldsymbol{\Phi q}$, equation (1) can be transformed to a state space model in modal basis:

$$
\begin{aligned}
& \dot{\mathbf{x}}=\mathbf{A x}+\mathbf{B u}=\left[\begin{array}{cc}
\mathbf{0} & \mathbf{I} \\
{\left[-\operatorname{diag}\left(\omega_{\mathrm{i}}^{2}\right)\right]} & {\left[-2 \operatorname{diag}\left(\xi_{\mathrm{i}} \omega_{\mathrm{i}}\right)\right]}
\end{array}\right]\left\{\begin{array}{c}
\mathbf{q} \\
\dot{\mathbf{q}}
\end{array}\right\}+\left[\begin{array}{c}
\mathbf{0} \\
\mathbf{\Phi}^{\mathrm{T}}
\end{array}\right] \mathbf{F} \\
& \mathbf{y}=\mathbf{C x}+\mathbf{D u}
\end{aligned}
$$

where $\omega_{1}$ are eigen frequencies and $\xi_{\mathrm{i}}$ modal damping ratios. A, B, C and $\mathbf{D}$ are dynamic, input, output and feedthrough matrix respectively. $\mathbf{u}$ is the control vector. The modal displacement vector $\mathbf{q}$ and the mode shape matrix $\boldsymbol{\Phi}$ can be reduced to $n$ modes, which are supposed to be sufficiently decoupled and the damping to be proportional and weak. $\Phi$ is normalized as:

$$
\boldsymbol{\Phi}^{\mathrm{T}} \mathbf{M} \boldsymbol{\Phi}=\mathbf{I}
$$

where $\mathbf{I}$ is identity matrix.

In this paper, the control algorithm is chosen to optimize the control gains from the modal model in equation (2). So, the classical Linear Quadratic (LQ) algorithm is used for realizing the modal control which can be updated through the change knowledge of $\mathbf{A}$ and $\mathbf{B}$ via the optimization is obtained thanks to Riccati's equations:

$$
\mathbf{S A}+\mathbf{A}^{\mathrm{T}} \mathbf{S}+\mathbf{Q}-\mathbf{S B R}^{-1} \mathbf{B}^{\mathrm{T}} \mathbf{S}=\mathbf{0}
$$

where $\mathbf{Q}$ and $\mathbf{R}$ are weighting matrices, $\mathbf{S}$ is the solution of the associated Riccati's equation. In this paper, $\mathbf{Q}$ and $\mathbf{R}$ are chosen to be constant and defined from the initial state of the structure. The optimal linear matrix gain $\mathbf{G}$ is obtained by the minimization of the energy quadratic performance function:

$$
J_{\min }=\int_{0}^{\infty}\left(\mathbf{x}^{\mathrm{T}} \mathbf{Q} \mathbf{x}+\mathbf{u}^{\mathrm{T}} \mathbf{R} \mathbf{u}\right) d t
$$

Then the optimal control is obtained as:

$$
\mathbf{u}=-\mathbf{G} \mathbf{x}
$$

where $\mathbf{G}=\mathbf{R}^{-1} \mathbf{B}^{\mathrm{T}} \mathbf{S}$ is the optimized matrix gain. 
The modal state vector $\mathbf{x}$ can't be obtained or measured directly. So it is estimated thanks to a Luenberger observer which delivers the modal state estimation $\hat{\mathbf{x}}$ :

$$
\dot{\hat{\mathbf{x}}}=\mathbf{A} \hat{\mathbf{x}}+\mathbf{B u}+\mathbf{L}(\mathbf{y}-\mathbf{C} \hat{\mathbf{x}}-\mathbf{D u})
$$

where $\mathbf{L}$ is the optimized observer gain.

\subsection{Identification of modal model}

In order to obtain the observer model A, B , C, D and compute the controller and observer gain matrix $\mathbf{G}$ and $\mathbf{L}$ from $\mathbf{A}, \mathbf{B}, \mathbf{C}, \mathbf{D}$, subspace method is chosen for getting a general state space model from input-output data. This identification method can be extended to closed-loop system as direct approaches [13] where an open-loop identification method is directly applied to identify the model of structure from the measured input signals which are necessary for the identification, the measured control signals, and response signals of the controlled structure. N4SID identification algorithm [18] is chosen for its convergence (noniterative method) and numerical stability, regardless of zero and non-zero initial states. By this algorithm, discrete state space matrices for time $k$ can be estimated by the least-squares method [13], with I/O data $\mathbf{u}$ and $\mathbf{y}$ :

$$
\left[\begin{array}{ll}
\mathbf{A}_{\mathrm{d}} & \mathbf{B}_{\mathrm{d}} \\
\mathbf{C}^{\prime} & \mathbf{D}^{\prime}
\end{array}\right]=\left(\left[\begin{array}{c}
\overline{\mathbf{X}}_{\mathrm{k}+1} \\
\overline{\mathbf{Y}}_{\mathrm{k} \mid \mathrm{k}}
\end{array}\right]\left[\begin{array}{c}
\overline{\mathbf{X}}_{\mathrm{k}} \\
\overline{\mathbf{U}}_{\mathrm{k} \mid \mathrm{k}}
\end{array}\right]^{\mathrm{T}}\right)\left(\left[\begin{array}{c}
\overline{\mathbf{X}}_{\mathrm{k}} \\
\overline{\mathbf{U}}_{\mathrm{k} \mid \mathrm{k}}
\end{array}\right]\left[\begin{array}{c}
\overline{\mathbf{X}}_{\mathrm{k}} \\
\overline{\mathbf{U}}_{\mathrm{k} \mid \mathrm{k}}
\end{array}\right]^{\mathrm{T}}\right)^{-1}
$$

where $\overline{\mathbf{X}} \cdots, \overline{\mathbf{X}}_{\mathrm{k}}, \overline{\mathbf{U}}_{\mathrm{k} \mid \mathrm{k}}, \overline{\mathbf{Y}}_{\mathrm{k} \mid \mathrm{k}}$ are the estimates of state vector, measured input and output vectors after time $k . \mathbf{A}_{\mathrm{d}}, \mathbf{B}_{\mathrm{d}}, \mathbf{C}^{\prime}, \mathbf{D}^{\prime}$ are the state space discrete identified matrices. Then classical method like matrix logarithm is used to transform this identified discrete state space matrices $\mathbf{A}_{\mathrm{d}}, \mathbf{B}_{\mathrm{d}}$ into continuous state space matrices $\mathbf{A}_{\mathrm{c}}, \mathbf{B}_{\mathrm{c}}$ and get the eigen value to deduce $\omega_{\mathrm{i}}$ and $\xi_{\mathrm{i}}$. But since the state space basis is unspecified, $\mathbf{A}_{\mathrm{c}}, \mathbf{B}_{\mathrm{c}}$ can not be directly used to reconstruct complex mode of structure, which is needed to get mode shape in equation (2). 
According to the different positions of actuator and sensor with at least one collocated pair, a transformation matrix $\boldsymbol{\tau}$ can be got from eigen vectors $\boldsymbol{\varphi}$ of $\mathbf{A}_{\mathrm{c}}$ by [16]:

$$
\mathbf{C}^{\mathrm{E}}(l,:) \boldsymbol{\varphi \tau ^ { 2 }}=\left(\boldsymbol{\varphi}^{-1} \mathbf{B}_{\mathrm{c}}^{\mathrm{E}}(:, l)\right)^{\mathrm{T}}
$$

where $l$ is the node of co-located actuator and sensor pair. $\mathbf{B}_{\mathrm{c}}^{\mathrm{E}}$ and $\mathbf{C}^{\mathrm{E}}$ are the expanded versions of $\mathbf{B}_{\mathrm{c}}$ and $\mathbf{C}^{\prime}$. Based on $\boldsymbol{\tau}$, complex modes of the structure $\boldsymbol{\psi}$ can be calculated from $\boldsymbol{\varphi}$. As mode shapes are supposed to be sufficiently decoupled and damping is supposed to be weak, a mode shape $\boldsymbol{\Phi}_{\mathrm{j}}$ can be estimated by [19]:

$$
\boldsymbol{\Phi}_{\mathrm{j}}=\boldsymbol{\Psi}_{2 \mathrm{j}} \sqrt{2 i \omega_{\mathrm{j}} \sqrt{1-\xi_{\mathrm{j}}^{2}}}, j=1 \cdots N
$$

where $\Psi_{2 \mathrm{j}}$ is the complex mode corresponding to eigen value $\lambda_{2 \mathrm{j}}=-\xi_{\mathrm{j}} \omega_{\mathrm{j}}+i \sqrt{1-\xi_{\mathrm{j}}^{2}} \omega_{\mathrm{j}}$ which is obtained from the identified continuous state space model. It must be noticed that equation (10) implies that $\boldsymbol{\Phi}_{\mathrm{j}}$ is normalized as in equation (3).

\subsection{Principle of self-adaptive modal control}

In this approach, the time-varying structure is controlled by the help of a feedback control loop combining an identifier. This principle is described in Figure1: output signals from the time-varying structure (a) are compared with those of the initial or previous identified model by a criterion (b). According to this criterion, if this model is too far from the actual system, an online modal parameter identification (c) is carried out to identify this structure. The identifier needs three types of signal: a random signal $r$ chosen for an efficient identification and the control input $u$ as excitations, output data $y$ as response signals of the controlled structure. This identified model (d) is used for updating the model of the observer (i) and also to optimize and update the observer gains (h) and controller gains (g). The controller and observer gains are optimized thanks to LQ (e) and LQG (f) algorithms with the updated modal state space model and this model can be also used in criterion (b). 
The updating time period of the self-adaptive control must be linked to the rate of the structure changes and to the first eigen frequency of the structure at initial state for obtaining good identification performances [20]. Moreover the control step time is defined from the highest frequency to be controlled. The weighting optimization matrices of the controller and observer are defined from the initial model of the structure and are kept for the following updates of the control system.

\subsubsection{Excitation for identifying the model}

In this type of identification chosen for a modal updating, the identifier is supplied with an I/O data directly connected to the structure. These input and output data are correlated due to the feedback control. In order to balance this problem, the relation between signal for identification and the disturbance should be considered as follows.

A white noise signal $r$ is added to the input signal $u$ (Figure.1). The level of this white noise signal $r$ is chosen in order to get an efficient identification and not to disturb the performance of the control. This level is also chosen to be greater than the environmental disturbance noise introduced on the structure as $v$ in Figure.1. Since this paper focuses on the simulation, the level of the disturbance noise $v$ can be defined but remains realistic. Then the level ratio $\alpha$ defined as:

$$
\alpha=\frac{\int_{0}^{t} r^{2} d t}{\int_{0}^{t} v^{2} d t}
$$

is considered as the level of energy between the identification signal $r$ and the disturbance noise $v$. So, $\alpha$ must be sufficiently large to ensure a correct identification.

The correlation of input and output data can be reflected by a correlation between the input signal $u+r$ and the response signal $y$, as covariance function $c_{\alpha}=\operatorname{Cov}(u+r, y)$. If $c_{\alpha} \approx 0$, the correlation is weak and results of identification are considered to be accurate. Therefore, $\alpha$ has to be sufficiently low to ensure that the correlation of signals is weak and 
acceptable. For different structure, some simulation or experiment is needed to find an optimal $\alpha$ for which $c_{\alpha}$ can be close to zero and the identification is effective in spite of disturbance noise. In addition, the disturbances considered in this paper are assumed not to modify the operating dynamic behaviour in a persistent manner.

\subsubsection{Criterion for identification}

Firstly, a systematic online real-time identification is proposed. The first step of the process is the collection of I/O data with a correct rate to describe the highest frequency mode. When the collection is large enough to describe the lowest frequency mode in order to get a correct state space model [20], the identification is carried out to update modal model and consecutively the controller and observer. This iterative procedure is then performed on new I/O data without overlapping points over the identification windows. This method is well adapted in the case of slow and smooth changes in the structure behaviour.

But for actual control system, identification should be carried out only when the initial or previous identified model is far from the current and actual behaviour. Comparing with the above proposed systematic online real-time identification which is taken as reference, identification with a criterion can be proposed. This criterion can be used both to trigger the identification and to update the control system.

For example, Modal Assurance Criterion (MAC) [21] has been proposed over the last twenty years to determine the exactness of identified mode shape. But for getting an updated mode shape which is combined with the previous mode shape to calculate the MAC value, a systematic identification is still needed. So the MAC can only be used as a criterion to trigger control changes.

The simplest way is to use a criterion based on the output signals of model and actual structure. For example the value of Variance-Accounted For (VAF) [17] is proposed. The VAF is defined as: 


$$
\operatorname{VAF}(y, \hat{y})=\max \left\{1-\frac{\operatorname{var}(y-\hat{y})}{\operatorname{var}(y)}, 0\right\} \times 100 \%
$$

where $\hat{y}$ is an output obtained by simulating the identified model and $y$ is the corresponding output of the actual changing structure as shown in Figure.1. Then the trigger for running identification and updating the control system is given when the criterion of VAF (shown in Figure.1) is less than or equal to a threshold value qualifying the updating sensitivity.

\section{Application to time-varying structures}

\subsection{Description of structure with 3 pendulums}

A 3 DoF discrete structure is chosen to illustrate the previous process of self-adaptive modal control. The 3 pendulums' mechanical structure is presented in Figure. 2 where the $3^{\text {rd }}$ pendulum mass can be moved over the axial direction. The mass displacement permits eigen frequency shifts and an inversion of mode shapes. The characteristics of this structure are summarized in Table 1.

Table 1: Physical and modal characteristics of the structure (initial state)

\begin{tabular}{cccc}
\hline $\begin{array}{c}\text { Mass of pendulums } \\
(\mathrm{Kg})\end{array}$ & $\begin{array}{c}\text { Stiffness of } \\
\text { spring }(\mathrm{N} / \mathrm{m})\end{array}$ & $\begin{array}{c}\text { Length of stems } \\
(\mathrm{m})\end{array}$ & $\begin{array}{c}\text { Mass of stems } \\
(\mathrm{Kg})\end{array}$ \\
$\mathrm{m}_{1}=2.61$ & $\mathrm{k}_{1}=13182$ & $\mathrm{Lt}_{1}=0.414$ & $\mathrm{Mt}_{1}=0.305$ \\
$\mathrm{~m}_{2}=2.61$ & $\mathrm{k}_{2}=13182$ & $\mathrm{Lt}_{2}=0.414$ & $\mathrm{Mt}_{2}=0.305$ \\
$\mathrm{~m}_{3}=0.875$ & $\mathrm{k}_{3}=13182$ & $\mathrm{Lt}_{3}=0.431$ & $\mathrm{Mt}_{3}=1.205$ \\
\hline Modal damping ratios & \multicolumn{3}{c}{ Initial eigen frequencies } \\
$\xi_{1}=2.6 .10^{-3}$ & \multicolumn{2}{c}{$3^{\text {rd }}$ pendulum mass at the bottom location $)$} \\
$\xi_{2}=2.6 .10^{-3}$ & $\mathrm{f}_{1}=6.44 \mathrm{~Hz}$ \\
$\xi_{3}=2.6 .10^{-3}$ & $\mathrm{f}_{2}=17.5 \mathrm{~Hz}$ \\
\hline
\end{tabular}

\subsection{Instability induced by mode shape inversion}

In this example, a regulation problem is considered in which a controller is used to reject the non collocated disturbance applied on the structure. When the position of $3^{\text {rd }}$ pendulum mass changes, some minor frequency shifts will occur and the $2^{\text {nd }}$ mode shape will 
be inverted by the change of the sign of the $2^{\text {nd }}$ component of the $2^{\text {nd }}$ mode shape (see Figure.7c,d).

From equation (2), the dynamic behaviour of the structure can be expressed in modal coordinates with a state space form:

$$
\begin{aligned}
& \dot{\mathbf{x}}=\mathbf{A x}+\mathbf{B u}+\mathbf{Z} v \\
& \mathbf{y}=\mathbf{C x}+\mathbf{D u}+\mathbf{H} \mathbf{v}
\end{aligned}
$$

where $\boldsymbol{V}$ represents the unknown external disturbance and unknown environmental noise, $\mathbf{Z}$ and $\mathbf{H}$ are disturbance matrices. Combining equations(6), (7) and (13), the modal state space form of the controlled /observed structure is given by:

$$
\begin{gathered}
{\left[\begin{array}{c}
\dot{\mathbf{x}} \\
\dot{\mathbf{x}}-\dot{\hat{\mathbf{x}}}
\end{array}\right]=\left[\begin{array}{cc}
\mathbf{A}-\mathbf{B G} & \mathbf{B G} \\
\mathbf{0} & \mathbf{A}-\mathbf{L C}
\end{array}\right]\left[\begin{array}{c}
\mathbf{x} \\
\mathbf{x}-\hat{\mathbf{x}}
\end{array}\right]+\left[\begin{array}{c}
\mathbf{Z} \\
\mathbf{Z}-\mathbf{L H}
\end{array}\right] \mathbf{V}} \\
\mathbf{y}=\left[\begin{array}{ll}
\mathbf{C}-\mathbf{D G} & \mathbf{D G}
\end{array}\right]\left[\begin{array}{c}
\mathbf{x} \\
\mathbf{x}-\hat{\mathbf{x}}
\end{array}\right]+\mathbf{H} \mathbf{v}
\end{gathered}
$$

If the force of control is applied to the $2^{\text {nd }}$ stem and outputs are measured by the help of the accelerations on each stem, matrices $\mathbf{B}, \mathbf{C}$ and $\mathbf{D}$ are written as:

$$
\begin{aligned}
& \mathbf{B}=\left[\begin{array}{llll}
\mathbf{0}_{1 \times 3} & \Phi_{21} & \Phi_{22} & \Phi_{23}
\end{array}\right]^{\mathrm{T}} L_{\mathrm{c}} L_{\mathrm{f}} \\
& \mathbf{C}=\boldsymbol{\Phi}\left[-\operatorname{diag}\left(\omega_{\mathrm{i}}^{2}\right)-2 \operatorname{diag}\left(\xi_{\mathrm{i}} \omega_{\mathrm{i}}\right)\right] \\
& \mathbf{D}=\boldsymbol{\Phi}\left[\begin{array}{lll}
\Phi_{21} & \Phi_{22} & \Phi_{23}
\end{array}\right]^{\mathrm{T}} L_{\mathrm{c}} L_{\mathrm{f}}
\end{aligned}
$$

where $\Phi_{\mathrm{r}, \mathrm{s}}$ is the $r$ th component of $s$ th mode shape.

The poles of the observed and controlled structure are governed by $\mathbf{A}-\mathbf{L C}, \mathbf{A}-\mathbf{B G}$ respectively. For the controller, the inversion of mode shape involved in matrix $\mathbf{B}$ is effective when the control force is applied to the $2^{\text {nd }}$ stem and then affects the poles of the controller. For the observer, the inversion of mode shape is always involved in its poles location.

The control gains and the observer gains $\left(\mathbf{G}_{0}, \mathbf{L}_{0}\right)$ are adjusted by LQG algorithm with weighting matrices $\mathbf{Q}$ and $\mathbf{R}$ chosen in order to get a high performance when $3^{\text {rd }}$ pendulum 
mass is located at the bottom location. Thanks to initial robustness and damping of controlled structure, the structure is stable before $\mathrm{Lm}_{3}=0.28 \mathrm{~m}$. But with this control adjustment $\left(\mathbf{G}_{0}, \mathbf{L}_{0}\right)$ when the $3^{\text {rd }}$ pendulum is moved from the bottom to the top, the controlled structure becomes unstable since the mass location is less than $0.29 \mathrm{~m}$ as shown in Figure.3. This instability is clearly due to the mode shape inversion as mentioned in [22].

What's more for an actual time-varying structure, the changes of modal characteristics like mode shape inversion can not be known a priori and measured. Therefore the real-time identification is necessary to update modal model and consecutively the controller.

\subsection{Self-adaptive control implementation}

This section is devoted to the validation of the updating principle of the controller and observer gains, of the observer model. In a first subsection, the changes of gains and model are expected to be safe and do not introduce some abrupt changes when a systematic updating process is chosen. Then, the updating scheme is improved by introducing a criterion on outputs which triggers the identification and the changes in the controlled structure. The last investigation focuses on the speed of the mass displacement in order to validate the robustness of VAF identification with external noise and disturbance. Acceleration and force signals are sampled at $1000 \mathrm{~Hz}$ and filtered, focusing the frequency bandwidth of interest to $[2 \mathrm{~Hz}$ $50 \mathrm{~Hz}]$. The length of the identification window is set to 500 points (see justifications on the same device in [20]).

\subsubsection{Validation of the updating principle}

In this section, the same weighting matrices $\mathbf{Q}$ and $\mathbf{R}$ as in section 3.2 are used by LQG to optimize the controller and the observer. By contrast with section 3.2, when the location of $3^{\text {rd }}$ mass is changed, the gains $\mathbf{G}$ (of the controller) and $\mathbf{L}$ (of the observer) and the observer model are updated according to the identified modal model $(\mathbf{A}, \mathbf{B}, \mathbf{C}, \mathbf{D})$. This update is carried out systematically without any criterion. The updating time between two 
successive changes is set to $0.5 \mathrm{~s}$ in accordance with the frequency bandwidth and the speed of the moving mass. This self-adaptive control is firstly tested in quasi static behaviour (in fact very slow motion conditions: mass velocity of $0.71 \mathrm{~mm} / \mathrm{s}$ ). These successive poles of observed and controlled systems are presented in Figure 4 and can be considered almost as same as estimations at different fixed locations of $3^{\text {rd }}$ mass. Like in section 3.2 , the poles of the controller move to the right, but after $\mathrm{Lm}_{3}=0.29 \mathrm{~m}$, the poles move back to the left due to the updating process. All the poles remain in the left side of complex plane with enough damping. So the stability of observed and controlled structure is then guaranteed.

Moreover, the performed modifications in spite of one state to the other are sufficiently continuous to guarantee that the effect of control is smooth and continuous in the updated time as shown in Figure 5a,b. In [65.0s-65.5s], a peak value of control force is induced by a bad precision of the identified model due to the weak magnitude of response signals near the mode shape inversion which occurs at $67.09 \mathrm{~s}$.

\subsubsection{Alternative updating by criterion}

In order to verify the ideal performances of conditional identification, firstly disturbance and environmental disturbance noise $v$ are not applied to the structure and the $3^{\text {rd }}$ mass moves also from $\mathrm{Lm}_{3}=0.342 \mathrm{~m}$ to $\mathrm{Lm}_{3}=0.234 \mathrm{~m}$ with the velocity of $3.6 \mathrm{~mm} / \mathrm{s}[20]$. Since $\mathbf{G}$ and $\mathbf{L}$ are updated according to the identified modal model, the control system is self-adapted to the changing structure. The threshold value of VAF for the $1^{\text {st }}$ pendulum acceleration is set to 95\% under which identification is carried out. The calculation of VAF is assumed to be carried out on a large enough window with the same rate as systematic identification taken as reference for comparison (i.e. 500 points sampled at $1000 \mathrm{~Hz}$ as shown in Figure.6).

With these operating conditions, $\Phi_{\mathrm{r}, \mathrm{s}} / \Phi_{1, \mathrm{~s}}$ and frequencies are identified by systematic and VAF identification as modal characteristics and compared to their theoretical values from the simulated structure. The values of VAF and some identified results as $\Phi_{2,2} / \Phi_{1,2}$ and 
frequency $\omega_{2}$ are shown in Figure 7a-d. For the 3 pendulums' mechanical structure, the $2^{\text {nd }}$ mode is of significance, so only the critical characteristics of the $2^{\text {nd }}$ mode are presented, $1^{\text {st }}$ and $3^{\text {rd }}$ modal characteristics being always identified with a precision under $5 \%$.

In the Figure 7, some instants when the difference between identified and theoretical response is large enough to produce a value of $\mathrm{VAF} \leq 95 \%$ are reported. At these instants the identification is carried out to update the characteristics of modal model of the structure, leading or not to an improvement of VAF criterion. In the Figure $7 \mathrm{~b}$, the second eigen frequency values are presented in comparison with systematic identification. As mentioned before, the value of VAF is calculated only on the first pendulum acceleration. Therefore attention must be paid to this global indicator takes into account all the modal characteristic changes, but only the second mode characteristics are presented. It must be noticed that this indicator is sufficiently sensitive to ensure robustness of the modal adaptive control of the proposed application.

At time $\mathrm{t}=3 \mathrm{~s}$, the initial model is not coincident with the structure, the difference on $\Phi_{2,2} / \Phi_{1,2}$ is great than $20 \%$ as shown in Figure $7 \mathrm{~d}$ (the other $\Phi_{\mathrm{r}, \mathrm{s}} / \Phi_{1, \mathrm{~s}}$ are less than $5 \%$ ) and the VAF is $92.12 \%$. Then identification is carried out and the model and the gains are updated. At time $\mathrm{t}=3.5 \mathrm{~s}$, there is still some difference between identified and simulated results during [3s-3.5s], but not mainly on the second mode characteristics. Again an identification and a model update are carried out again at time $\mathrm{t}=3.5 \mathrm{~s}$ and at time $\mathrm{t}=4 \mathrm{~s}$. As the updated value of VAF is $97.79 \%$ at time $\mathrm{t}=4.5 \mathrm{~s}$, the identification and the updating process are stopped.

After time $t=13.42 \mathrm{~s}$, instant when the inversion of mode shape occurs, the value VAF decreases suddenly as depicted in Figure 7a. About eight identification steps are necessary to recover a VAF larger than $95 \%$. This means that a mode shape inversion is associated with a difficulty to recover a modal model of this particular structure. Indeed, when the $2^{\text {nd }}$ mode shape is close to be inverted, i.e $\Phi_{2,2} \approx 0$, the acceleration of $2^{\text {nd }}$ pendulum mass is near to 
zero. Then the identifier can not get enough informative outputs leading to bad identified modal characteristics. A full study devoted to this problem is out of the scope of the present paper.

When the inversion occurs, the VAF identified model is less exact than systematically identified model, but is sufficient to update the controller and the observer, therefore improving the efficiency of control system. During the movement of $3^{\text {rd }}$ mass, the necessary noise for the identification leads to unwanted displacement and controlled force. As shown in Figure 8a-b as an example, the displacement $x_{3}$ and the controller force are smooth, weak and stable over the time interval. So, this self-adaptive control system yielding abrupt changes in model and gains does not introduce instability.

\subsubsection{VAF identification robustness}

The effect of VAF identification is affected by the speed of variation of time-varying structure. For this 3 DoF structure, this variation is governed by the velocity of the $3^{\text {rd }}$ pendulum mass. To investigate this influence on the identified results, a velocity shape is used for simulation and shown in Figure 9a. By this shape, the changes on the identified results induced by velocity and by mode shape inversion can be distinguished. The resulting position of the $3^{\text {rd }}$ pendulum is moved from $\mathrm{Lm}_{3}=0.342 \mathrm{~m}$ to $\mathrm{Lm}_{3}=0.230 \mathrm{~m}$ as shown in Figure $9 \mathrm{~b}$.

All the other conditions are the same as in section 3.3.2 and corresponding values of VAF and identified modal characteristics are shown in Figure 10a-c.

Due to a higher velocity of $6.5 \mathrm{~mm} / \mathrm{s}$, the initial model is not coincident with the structure at time $t=2 \mathrm{~s}$ as shown in Figure $10 \mathrm{c}$, so the first detection with the VAF criterion, the identification and the update process occur earlier than that in section 3.3.2.

Before the mode shape inversion occurring at time $t=9.13 \mathrm{~s}$, the smallest value of VAF is $29.48 \%$ at time $\mathrm{t}=6 \mathrm{~s}$ (Figure $10 \mathrm{a}$ ), as it's $63.38 \%$ with constant velocity of $3.6 \mathrm{~mm} / \mathrm{s}$ (Figure 7a). This is induced by: 
(a) the velocity is $4.5 \mathrm{~mm} / \mathrm{s}$ (Figure $9 \mathrm{a}$ ) which is great than $3.6 \mathrm{~mm} / \mathrm{s}$.

(b) the position of $3^{\text {rd }}$ pendulum is $0.306 \mathrm{~m}$, more close to the position of inversion than that of constant velocity $3.6 \mathrm{~mm} / \mathrm{s}$, as shown in Figure $9 \mathrm{~b}$ by the two red points.

Close to the time of mode shape inversion, the velocity is $2.94 \mathrm{~mm} / \mathrm{s}$ which is less than the constant velocity $3.6 \mathrm{~mm} / \mathrm{s}$, so the identified model (see Figure 10b-c) is more precise than results presented in section 3.3.2 (Figure 7b-c ). After the mode shape inversion, the value of VAF is restored to $95 \%$ after a longer time (16 identification steps) due to lower velocity in this time period. With a lower velocity, the $3^{\text {rd }}$ pendulum mass spends a long time to leave the position of inversion. When the mass velocity reaches $0 \mathrm{~m} / \mathrm{s}$ at time $\mathrm{t}=15 \mathrm{~s}$, the identified results are almost equal to the theoretical values. After time $t=25 \mathrm{~s}$ when velocity reaches $5 \mathrm{~mm} / \mathrm{s}$, the identification occurs more frequently.

The sample time and the length of the identification window are constant and obviously the variation speed of time-varying structure will affect its dynamic behaviour. If the speed is null, then the accuracy is optimal. In the case of mass movement, the accuracy of the identification depends on the magnitude of the change of dynamic behaviour in this identification window, so depends on the mass speed. And a trade-off between update frequency and exactness of identified modal model must be found by setting the VAF threshold value.

As described in section 3.3.2, the displacement $x_{3}$ and the controller force remain always smooth, weak and stable. The VAF identification is robust since it offers the possibility to adjust the density of update and give a suitable identified model, according to different levels of variation of time-varying structure.

\subsection{Results of self-adaptive modal control}

From the above results, the self-adaptive control has been shown to work properly, but an updated control system has to be efficient to reject some disturbances on the structure. As 
the disturbance does not affect the dynamic behaviour, the identification process is expected to be not seriously affected by this disturbance. For getting changes of identified results induced by disturbance, a impulsive force of $50 \mathrm{~N}$ as an example of the unknown disturbance is applied at the end of $3^{\text {rd }}$ stem at arbitrary time $\mathrm{t}=20.22 \mathrm{~s}$ and during $20 \mathrm{~ms}$. This time location is chosen to clearly distinguish effects of disturbance from changes induced by mode shape inversion. The environmental white noise $v$ is also introduced in the system in order to simulate external disturbance noise. For this structure, $\alpha \geq 10^{6}$ is chosen to get $c_{\alpha} \approx 0$. Other operating conditions are kept the same as in section 3.3.2. The same parameters are tracked (value of VAF, identified $\Phi_{2,2} / \Phi_{1,2}$, and frequency $\omega_{2}$ ) and shown in Figure 11a-c.

For the value of VAF reported in Figure 11a, before the disturbance occurs, it is almost the same value as in section 3.3.2. As the disturbance induces changes on responses in the time interval $[20 \mathrm{~s} ; 20.5 \mathrm{~s}]$, the value of VAF decreases at time $\mathrm{t}=20.5 \mathrm{~s}$. As the force of control is applied as input of the model which is used to calculate $\hat{y}$, but the force of control applied on the structure is used to reject the pulse force, so the value of VAF remains close to zero more than $40 \mathrm{~s}$ after the disturbance. After time $\mathrm{t}=64.5 \mathrm{~s}$, VAF reaches again the threshold value of $95 \%$. In comparison with the mode shape inversion, an unknown disturbance induces higher difficulties to overcome for having a precise identified model.

From Figure 11b-c, comparison between systematic identification and VAF identification shows a very small difference between the two proposed approaches. Obviously, near the time $t=20.22 \mathrm{~s}$ where the disturbance occurs, there is a greater difference between theoretical and identified value of mode shape and frequencies, in time interval [20.5s-21s].

Even if the identified modal model is not exact during the time interval [20.5s-21.0s], thanks to updated gains and to the observer model, the controlled system remains stable. A high performance can be got to reject the effect of disturbance as shown in Figure 12a-b. 
So, self-adaptive modal control system with systematic or VAF identification is verified to be robust and to keep a high performance for changing structures with smooth and weak variation. And thorough VAF identification, the control system can demonstrate some smart propriety.

\section{Conclusion}

The aim of this paper is to propose a self-adaptive modal control including both identification and control update in real-time. This method is particularly suitable for timevarying structures. It can overcome the instability which is induced by the variation of modal characteristics, especially the inversion of mode shape. In the same time, this approach leads to a global robustness and keeps high performances given by control optimisation.

A classical LQG algorithm has been chosen here but the method can be extended to other control algorithms using a model of the structure. VAF method is employed as a criterion for determining the exactness of the model. Even if this criterion is only based on one response signal, it is demonstrated to be relevant for describing exactness of the identified model. This conditional identification represents an interesting alternative to modal comparison like MAC criterion which requires a systematic identification. For getting an updated model of the structure when behaviour changes occur, N4SID is used as a direct approach of identification of the structure under control to reach a modal state space model. Thanks to this identified model, the control system can be updated in agreement with the modal characteristics of the structure: frequency bandwidth and identification window length.

Through the simulation of a 3-DoF structure, some parameters like window length and sample frequency are governed by modal properties of the studied structure. Moreover, the VAF threshold is shown to be a good adjustment parameter to deal with the trade-off between exactness and frequency of updates, improving the efficiency of the self-adaptive control. The proposed identification process can be carried out in time domain and this method can exactly 
identify almost all modal characteristics with a $\pm 5 \%$ precision at least. Obviously when a modal inversion and/or a high and sudden disturbance occur, the exactness of identified mode shapes is reduced. But due to the updated control system, the stability can be guaranteed. Thorough this application, the self-adaptive modal control with VAF identification demonstrates a global robustness and some smart properties. In the same time, it allows to keep the high performance of optimized controls like LQG in spite of the changes of structure. Thanks to VAF criterion, this self-adaptive modal control system demonstrates some smart properties.

\section{References:}

[1] A. Preumont, B. De Marneffe and S. Krenk, "Transmission zeros in structural control with collocated multi-input/multi-output pairs," Journal of Guidance, Control, and Dynamics, vol. 31, 2008, p. 428-431.

[2] B. Chomette, D. Rémond, S. Chesné and L. Gaudiller, "Semi-adaptive modal control of on-board electronic boards using an identification method," Smart Materials and Structures, vol. 17, 2008, 8p.

[3] L. Gaudiller and J. Der Hagopian, "Active control of flexible structures using a minimum number of components," Journal of Sound and Vibration, vol. 193, 1996, p. 713-741.

[4] F. Matichard and L. Gaudiller, "Improvement of potential energetic exchange using non linear control," IEEE-ASME AIM - Advanced Intelligent Mechatronics,. Monterey, California, USA,2005, Monterey, California, USA: 2005, p. 807-812.

[5] L. Gaudiller and F. Matichard, "A nonlinear method for improving the active control efficiency of smart structures subjected to rigid body motions," IEEE/ASME Transactions on Mechatronics, vol. 12, 2007, p. 542-548. 
[6] I. Postlethwaite, M.C. Turner and G. Herrmann, "Robust control applications," Annual Reviews in Control, vol. 31, 2007, p. 27-39.

[7] R. Marino and P. Tomei, Nonlinear control design: Geometric, adaptive and robust, London: 1995.

[8] B.D. Anderson and A. Dehghani, "Challenges of adaptive control-past, permanent and future," Annual Reviews in Control, vol. 32, 2008, p. 123-135.

[9] B. Anderson, T. Brinsmead, F. De Bruyne, J. Hespanha, D. Liberzon and A. Morse, "Multiple model adaptive control. Part 1: Finite controller coverings," International Journal of Robust and Nonlinear Control, vol. 10, 2000, p. 909-929.

[10]L. Gaudiller and S. Bochard, “Adaptive active control of flexible structures subjected to rigid body displacements," Journal of Sound and Vibration, vol. 283, 2005, p. 311339.

[11]M. Gevers, "Identification for control: From the early achievements to the revival of experiment design,” European Journal of Control, vol. 11, 2005, p. 335-352.

[12]P.M. Van Den Hof and R.J. Schrama, "Identification and control- closed-loop issues," Automatica, vol. 31, 1995, p. 1751-1770.

[13]T. Katayama, Subspace methods for system identification, Springer-Verlag,London: 2005.

[14]M. Gilson and P.M. Van Den Hof, "Instrumental variable methods for closed-loop system identification," Automatica, vol. 41, 2005, p. 241-249.

[15]H. Hjalmarsson, "Iterative feedback tuning - An overview," International Journal of Adaptive Control and Signal Processing, vol. 16, 2002, p. 373-395.

[16]H. Luş, "Control theory based system identification," PhD.Thesis,Columbia University, New York, 2001. 
[17]J. Van Wingerden, I. Houtzager, F. Felici and M. Verhaegen, "Closed-loop identification of the time-varying dynamics of variable-speed wind turbines," International Journal of Robust and Nonlinear Control, vol. 19, 2009, p. 4-21.

[18]P. Van Overschee and B. De Moor, Subspace identification for linear systems: Theory implementation applications, Kluwer Academic Publishers, Dordrecht, Netherlands: 1996.

[19]E. Balmès, "New results on the identification of normal modes from experimental complex modes," Mechanical Systems and Signal Processing, vol.11,1997, p. 229-243.

[20]L. Renaudin and D. Rémond, "Modal Behavior Monitoring of a Discrete Evolutive Structure," Proceedings of the 15th IFAC symposium on System Identification, St Malo, France: 2009.

[21]R.J. Allemang, "The modal assurance criterion - twenty years of use and abuse," Sound and vibration, vol. 37, 2003, p. 14-23.

[22]F. Deng, D. Rémond and L. Gaudiller, "Modal control using real-time identification for time-varying structures," Proceedings of the biannual conference Vibration Shock and Noise (VCB), France, Écully, June 15-17, 2010. 
List of figure

Figure 1: Principle of self-adaptive modal control

Figure 2: The time-varying structure under study

Figure 3 In the case of non-updated controller $\mathbf{G}_{0}$ and observer $\mathbf{L}_{0}:$ (a) pole location

Figure 3 In the case of non-updated controller $\mathbf{G}_{0}$ and observer $\mathbf{L}_{0}:$ (b) details of pole location

Figure 4 In the case of updated controller $\mathbf{G}$ and observer $\mathbf{L}$ : (a) pole location

Figure 4 In the case of updated controller $\mathbf{G}$ and observer $\mathbf{L}$ : (b) details of pole location

Figure 5 Force of control when controller $\mathbf{G}$ and observer $\mathbf{L}$ are updated: (a) overall time history (mass motion velocity of $0.71 \mathrm{~mm} / \mathrm{s}$ ).

Figure 5 Force of control when controller $\mathbf{G}$ and observer $\mathbf{L}$ are updated: (b) details of some updated instants (mass motion velocity of $0.71 \mathrm{~mm} / \mathrm{s}$ ).

Figure 6: The time relation of VAF and identification

Figure $7 \mathrm{VAF}$ and identified modal characteristics during mass movement at $3.6 \mathrm{~mm} / \mathrm{s}$ : (a) VAF value change

Figure $7 \mathrm{VAF}$ and identified modal characteristics during mass movement at $3.6 \mathrm{~mm} / \mathrm{s}:(\mathrm{b})$ second frequency change

Figure 7 VAF and identified modal characteristics during mass movement at $3.6 \mathrm{~mm} / \mathrm{s}:$ (c) $2^{\text {nd }}$ component of $2^{\text {nd }}$ mode shape change

Figure $7 \mathrm{VAF}$ and identified modal characteristics during mass movement at $3.6 \mathrm{~mm} / \mathrm{s}$ : (d) details of $2^{\text {nd }}$ component of $2^{\text {nd }}$ mode shape change

Figure 8 In the case of updated controller $\mathbf{G}$ and observer $\mathbf{L}$ with VAF identification, and without disturbance: (a) displacement of $x_{3}$

Figure 8 In the case of updated controller $\mathbf{G}$ and observer $\mathbf{L}$ with VAF identification, and without disturbance: (b) force of control

Figure 9: (a) Velocity of the $3^{\text {rd }}$ pendulum mass

Figure 9: (b) displacement of the $3^{\text {rd }}$ pendulum mass

Figure 10 The value of VAF and identified results without disturbance under changing velocity of $3^{\text {rd }}$ pendulum mass: (a) VAF value change

Figure 10 The value of VAF and identified results without disturbance under changing velocity of $3^{\text {rd }}$ pendulum mass: (b) second frequency change 
Figure 10 The value of VAF and identified results without disturbance under changing velocity of $3^{\text {rd }}$ pendulum mass: (c) $2^{\text {nd }}$ component of $2^{\text {nd }}$ mode shape change

Figure 11 The value of VAF and identified results with disturbance: (a) VAF value

Figure 11 The value of VAF and identified results with disturbance: (b) second frequency change

Figure 11 The value of VAF and identified results with disturbance: (c) $2^{\text {nd }}$ component of $2^{\text {nd }}$ mode shape change

Figure 12 In the case of updated controller $\mathbf{G}$ and observer $\mathbf{L}$ with VAF identification, with disturbance: (a) displacement of $x_{3}$

Figure 12 In the case of updated controller $\mathbf{G}$ and observer $\mathbf{L}$ with VAF identification, with disturbance: (b) force of control 


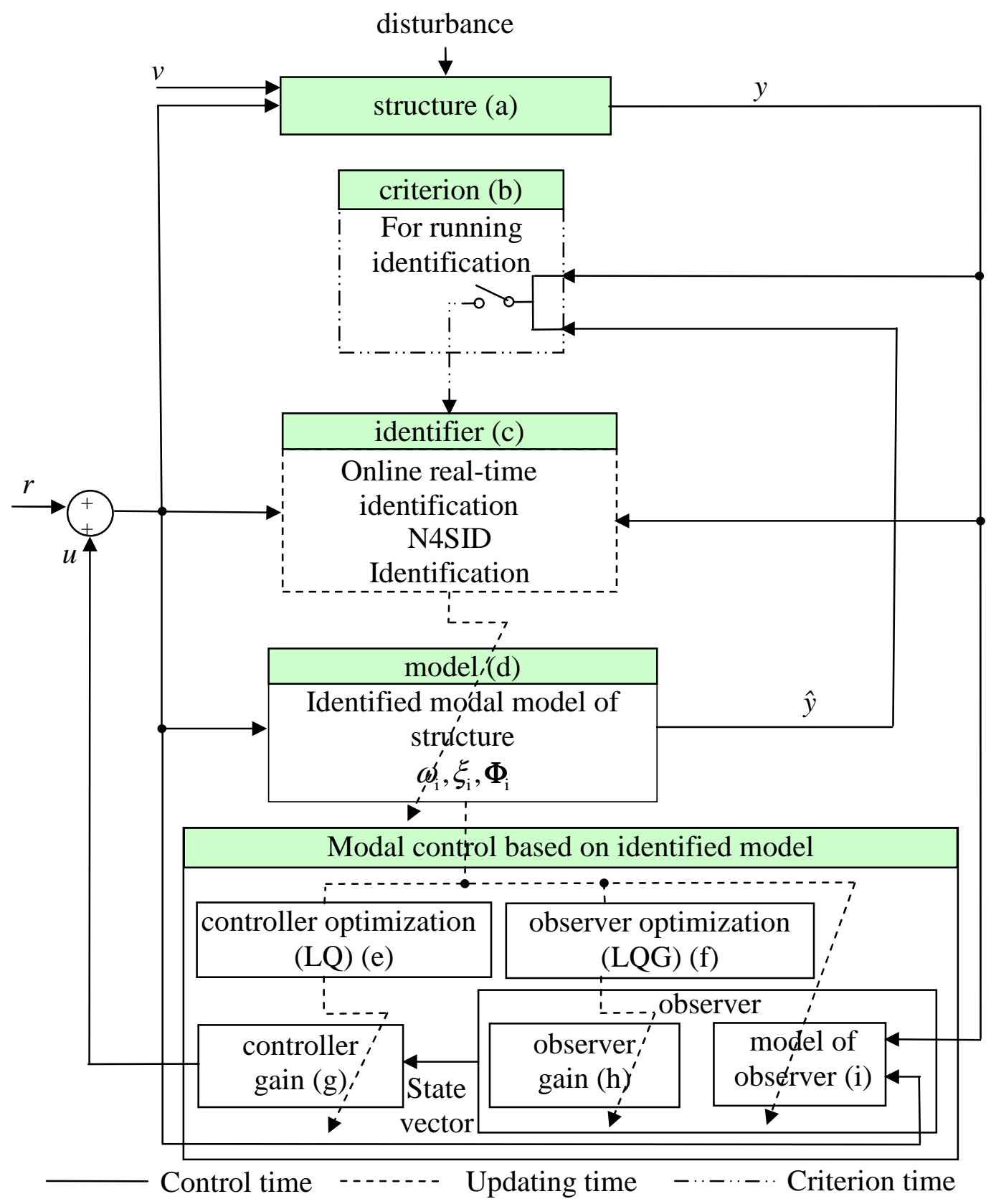

Figure 1: Principle of self-adaptive modal control 


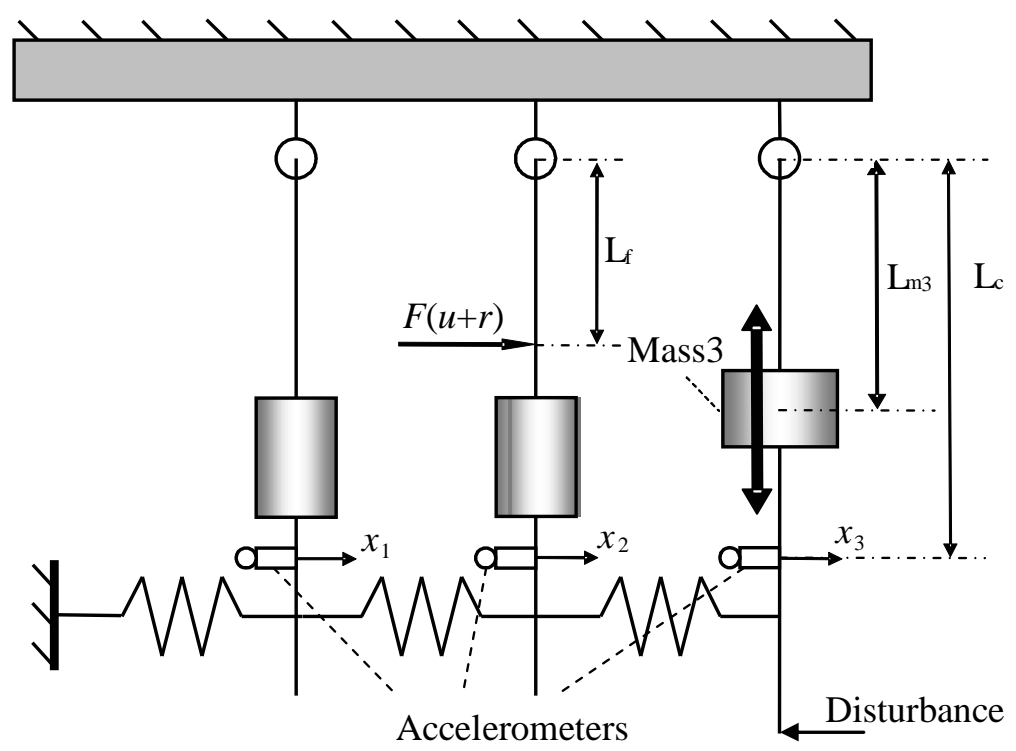

Figure 2: The time-varying structure under study 


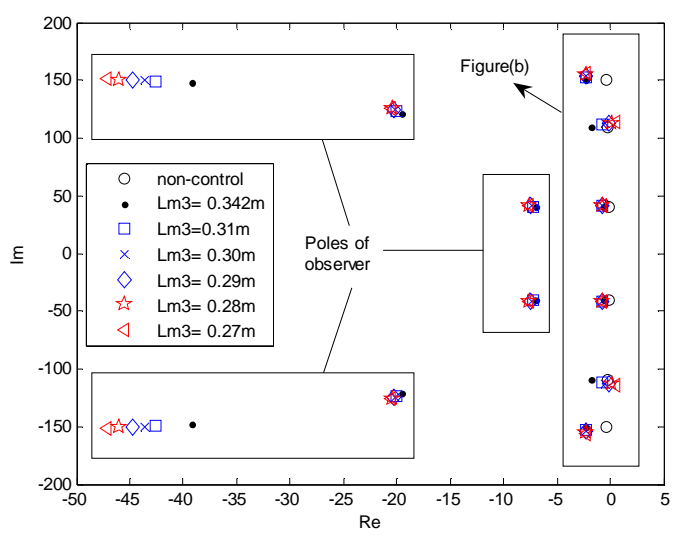

(a)

Figure 3 In the case of non-updated controller $\mathbf{G}_{0}$ and observer $\mathbf{L}_{0}$ : (a) pole location 


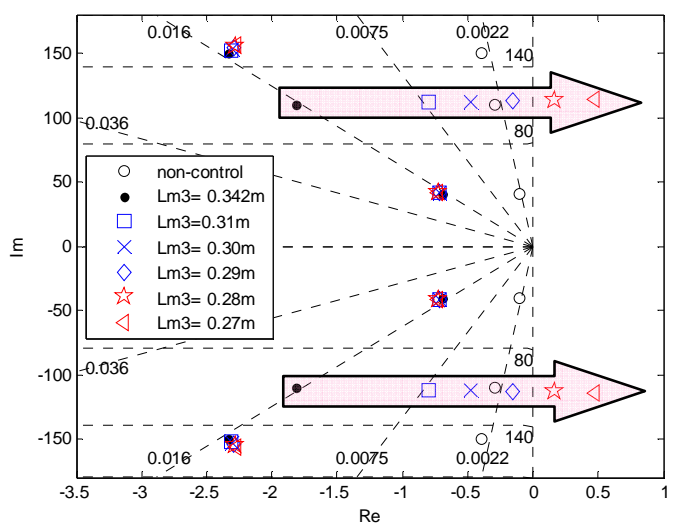

(b)

Figure 3 In the case of non-updated controller $\mathbf{G}_{0}$ and observer $\mathbf{L}_{0}:$ (b) details of pole location 


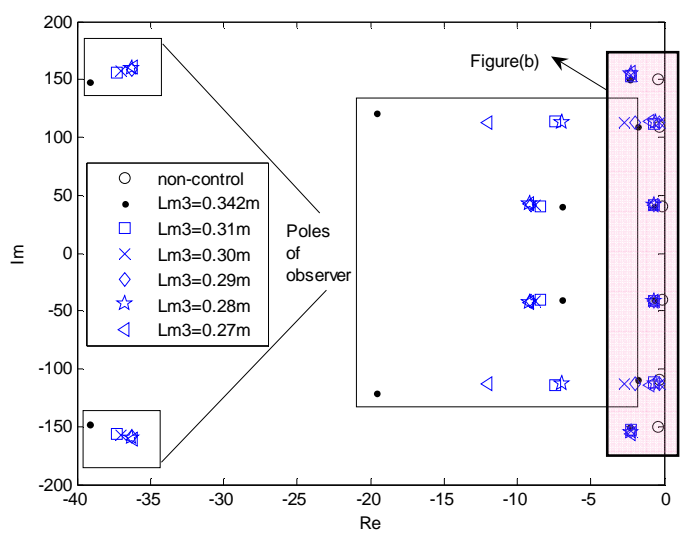

(a)

Figure 4 In the case of updated controller $\mathbf{G}$ and observer $\mathbf{L}$ : (a) pole location 


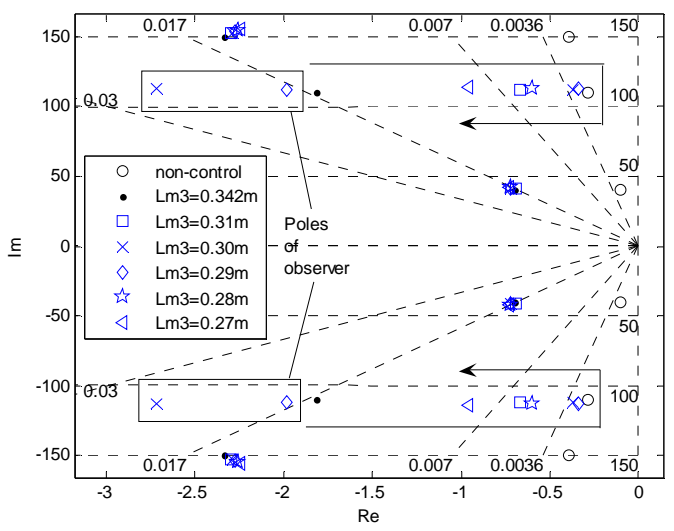

(b)

Figure 4 In the case of updated controller $\mathbf{G}$ and observer $\mathbf{L}$ : (b) details of pole location 


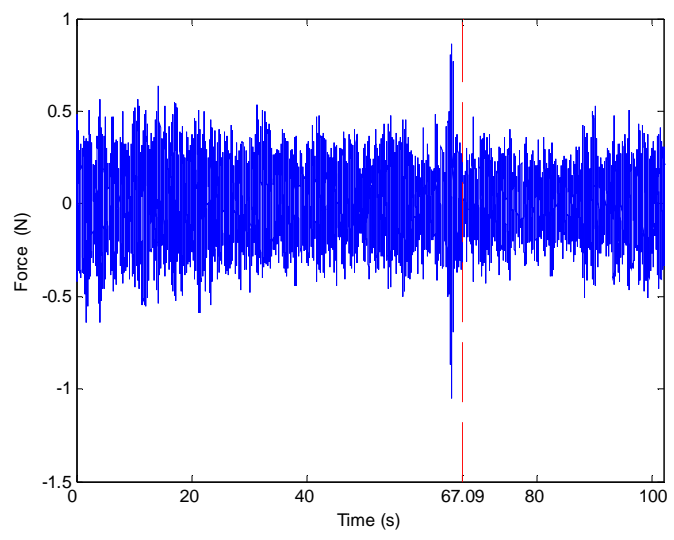

(a)

Figure 5 Force of control when controller $\mathbf{G}$ and observer $\mathbf{L}$ are updated: (a) overall time history

(mass motion velocity of $0.71 \mathrm{~mm} / \mathrm{s}$ ). 


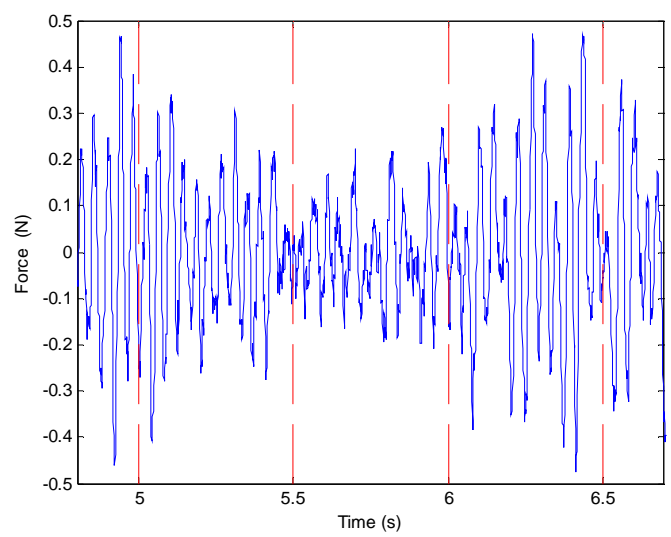

(b)

Figure 5 Force of control when controller $\mathbf{G}$ and observer $\mathbf{L}$ are updated: (b) details of some updated instants

(mass motion velocity of $0.71 \mathrm{~mm} / \mathrm{s}$ ). 


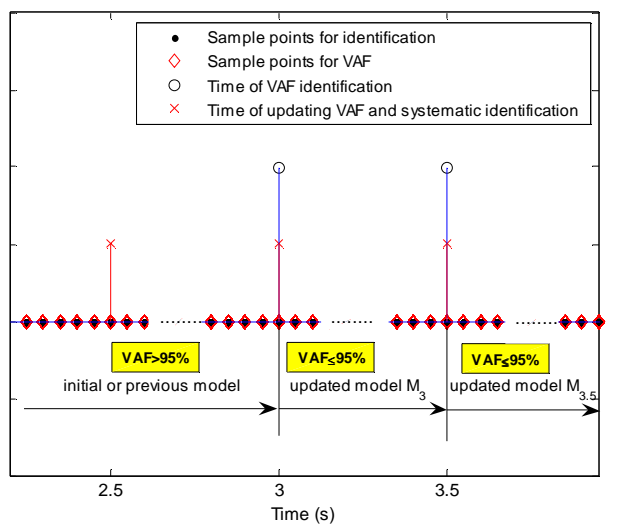

Figure 6: The time relation of VAF and identification. 


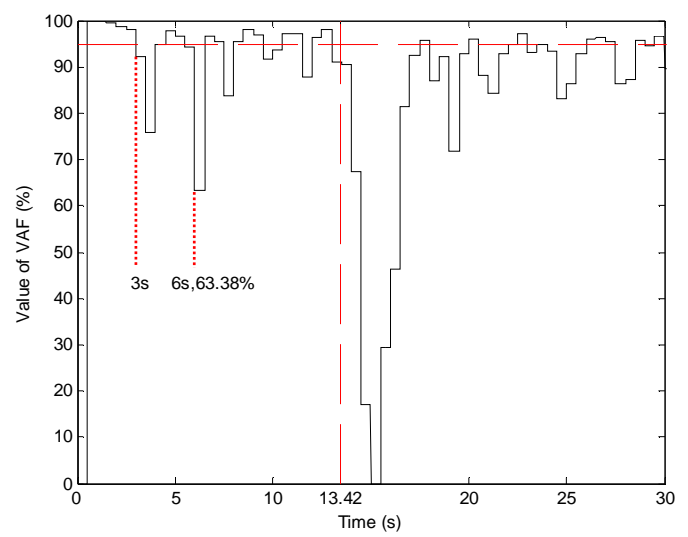

(a)

Figure 7: VAF and identified modal characteristics during mass movement at $3.6 \mathrm{~mm} / \mathrm{s}$ : (a) VAF value change 


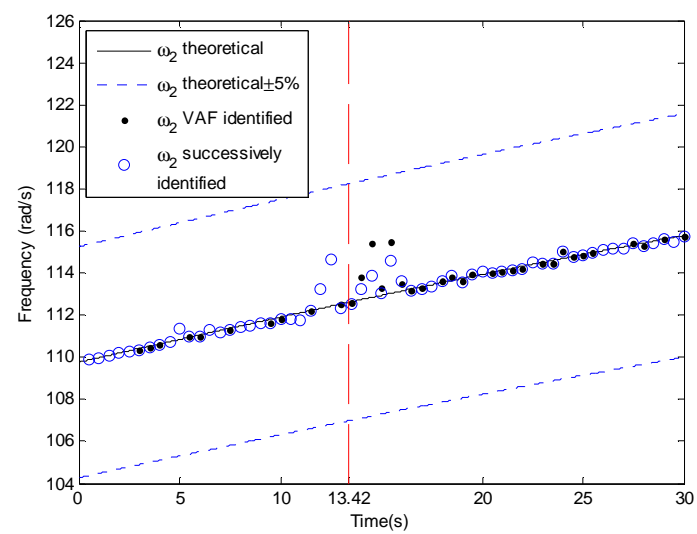

(b)

Figure 7: VAF and identified modal characteristics during mass movement at $3.6 \mathrm{~mm} / \mathrm{s}:$ (b) second frequency change 


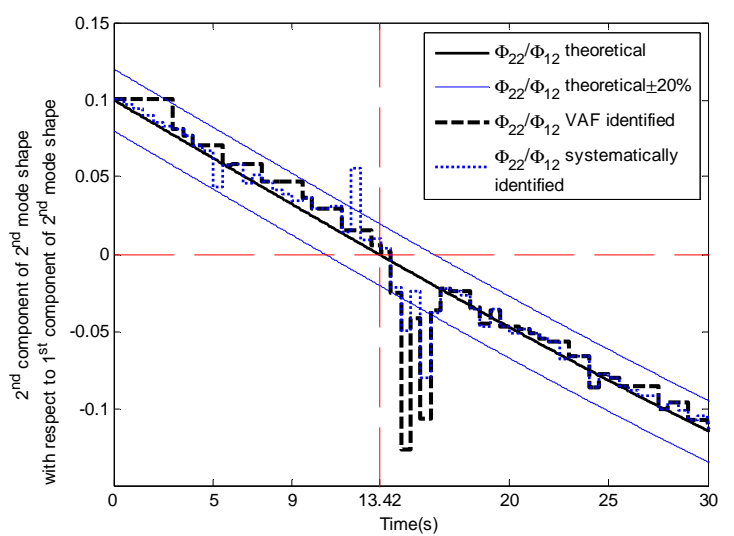

(c)

Figure 7: VAF and identified modal characteristics during mass movement at $3.6 \mathrm{~mm} / \mathrm{s}$ : (c) $2^{\text {nd }}$ component of $2^{\text {nd }}$ mode shape change 


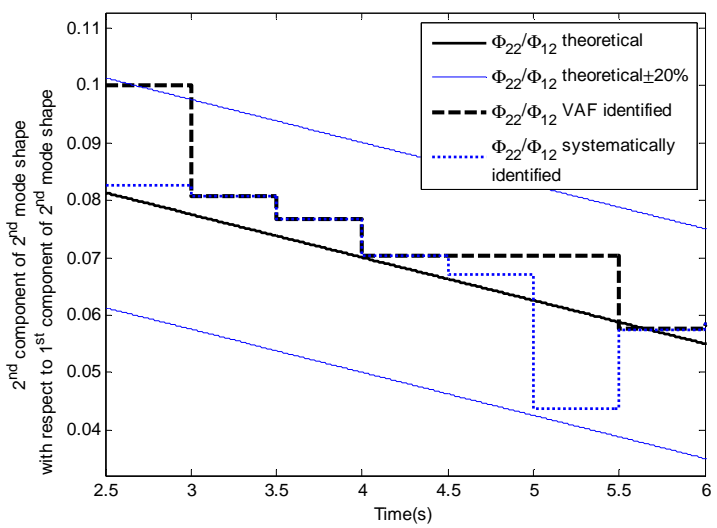

(d)

Figure 7: VAF and identified modal characteristics during mass movement at $3.6 \mathrm{~mm} / \mathrm{s}:(\mathrm{d})$ details of $2^{\text {nd }}$ component of $2^{\text {nd }}$ mode shape change 


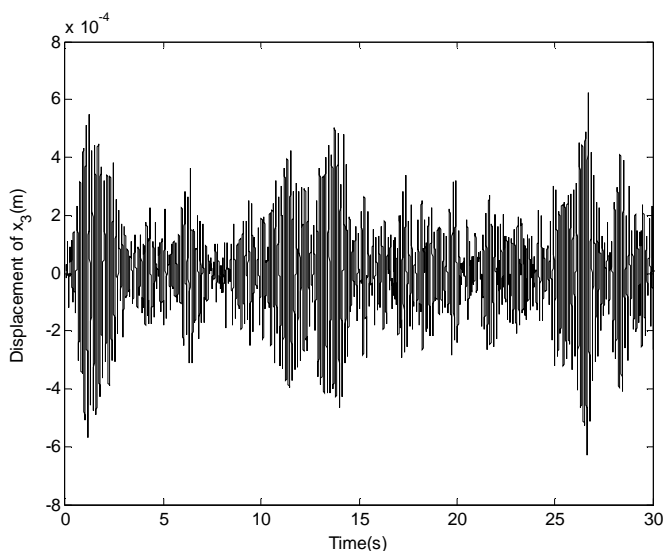

(a)

Figure 8: In the case of updated controller $\mathbf{G}$ and observer $\mathbf{L}$ with VAF identification, and without disturbance: (a) displacement of $x_{3}$ 


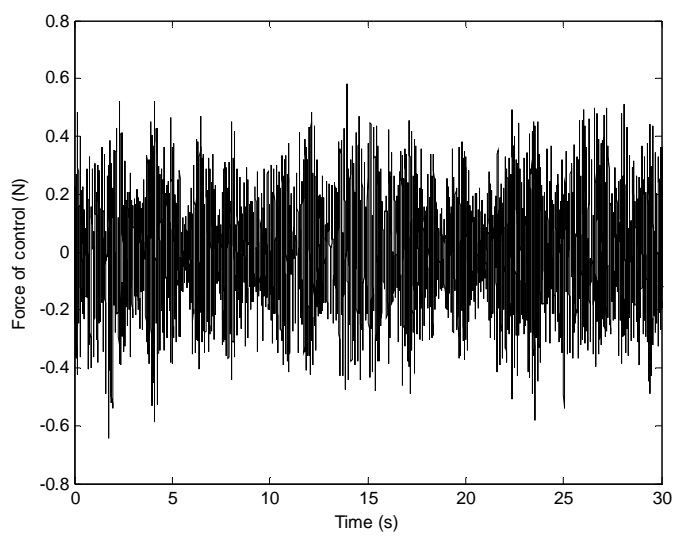

(b)

Figure 8: In the case of updated controller $\mathbf{G}$ and observer $\mathbf{L}$ with VAF identification, and without disturbance: (b) force of control 


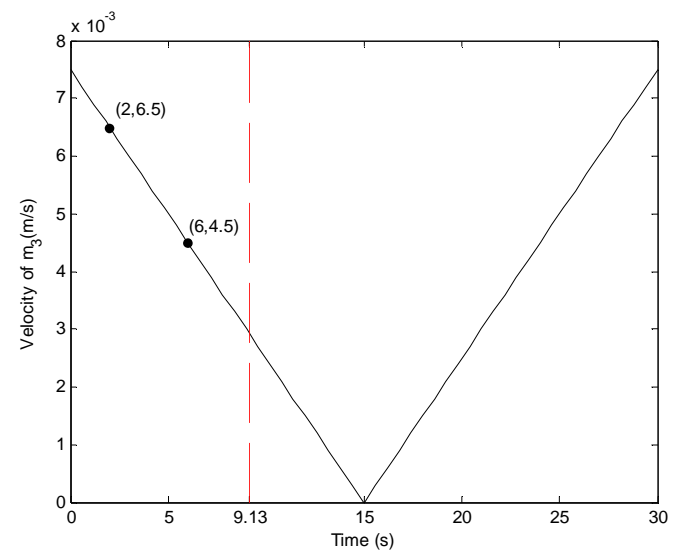

(a)

Figure 9: (a) Velocity of the $3^{\text {rd }}$ pendulum mass 


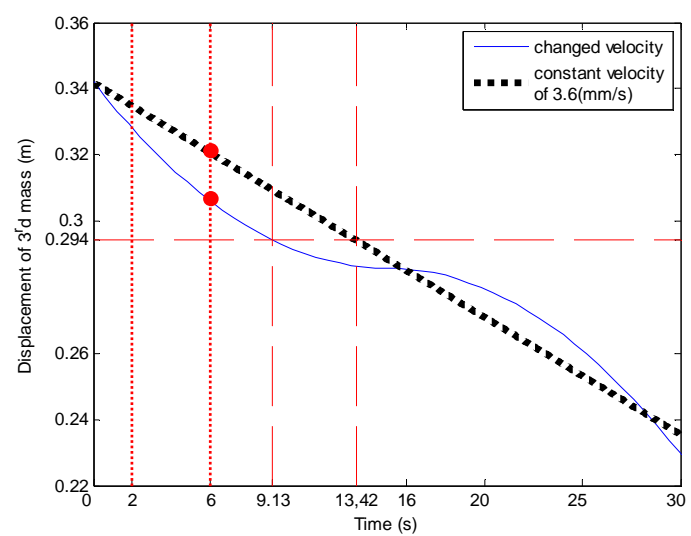

(b)

Figure 9: (b) displacement of the $3^{\text {rd }}$ pendulum mass 


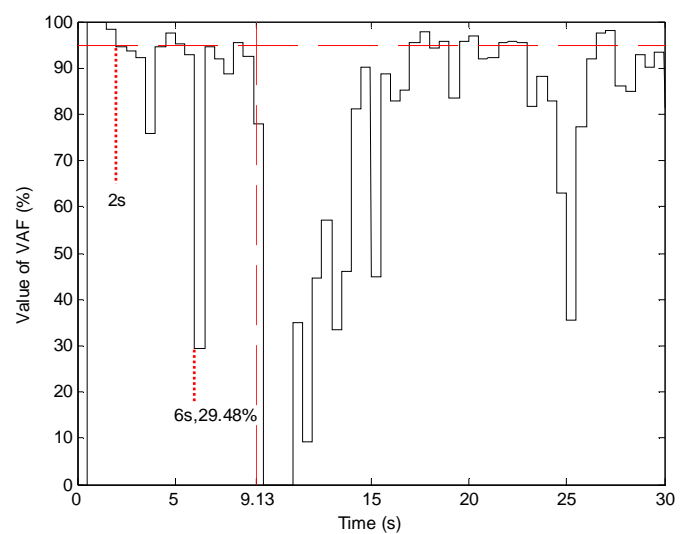

(a)

Figure 10 The value of VAF and identified results without disturbance under changing velocity of $3^{\text {rd }}$ pendulum mass: (a) VAF value change 


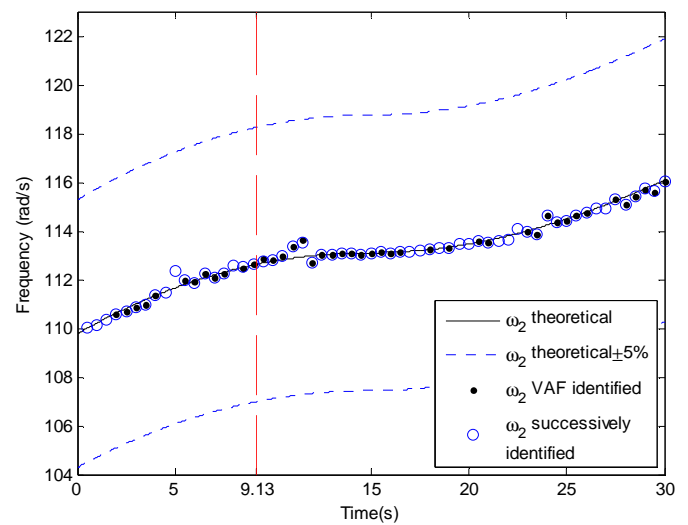

(b)

Figure 10 The value of VAF and identified results without disturbance under changing velocity of $3^{\text {rd }}$ pendulum mass: (b) second frequency change 


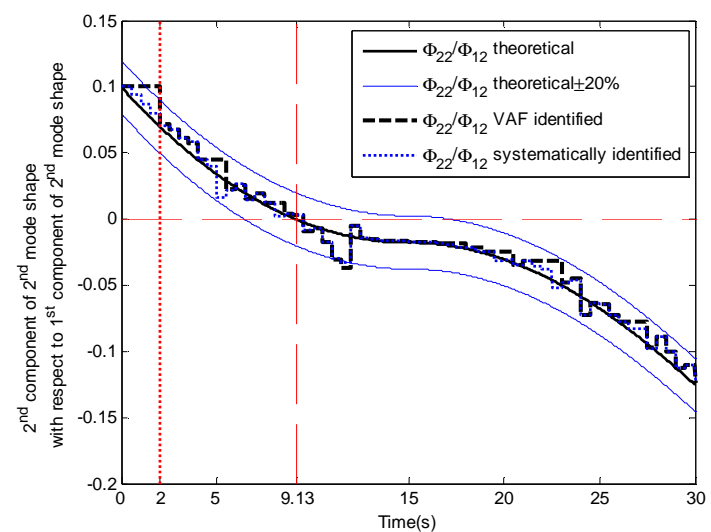

(c)

Figure 10 The value of VAF and identified results without disturbance under changing velocity of $3^{\text {rd }}$ pendulum mass: (c) $2^{\text {nd }}$ component of $2^{\text {nd }}$ mode shape change 


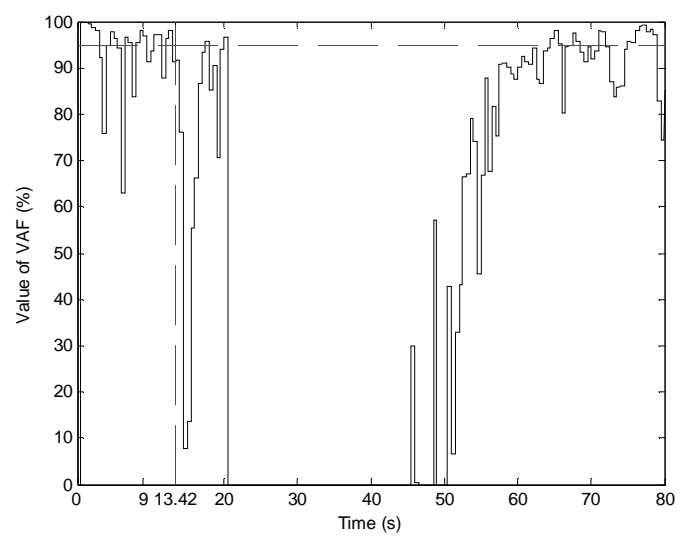

(a)

Figure 11 The value of VAF and identified results with disturbance: (a) VAF value 


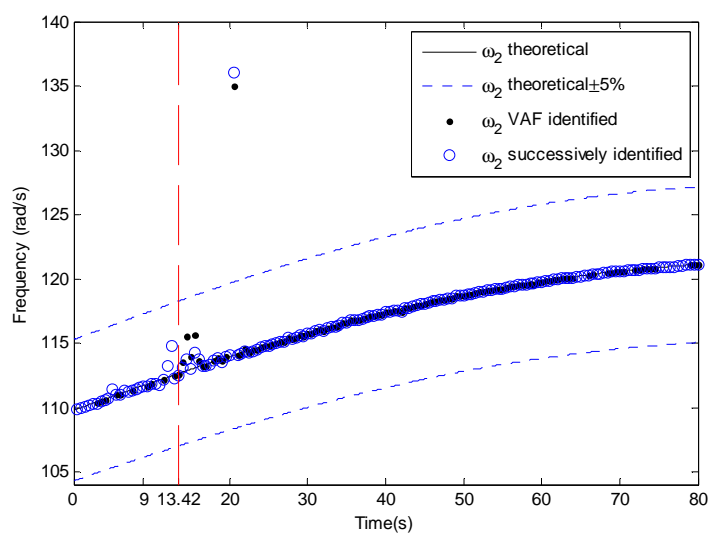

(b)

Figure 11 The value of VAF and identified results with disturbance: (b) second frequency change 


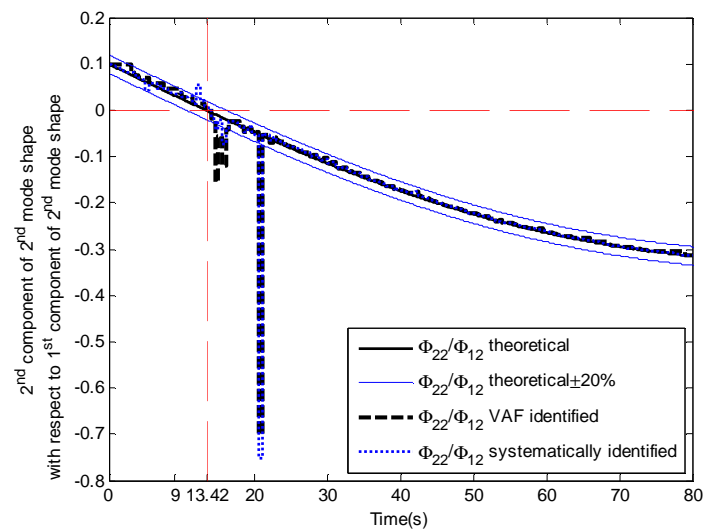

(c)

Figure 11 The value of VAF and identified results with disturbance: (c) $2^{\text {nd }}$ component of $2^{\text {nd }}$ mode shape change 


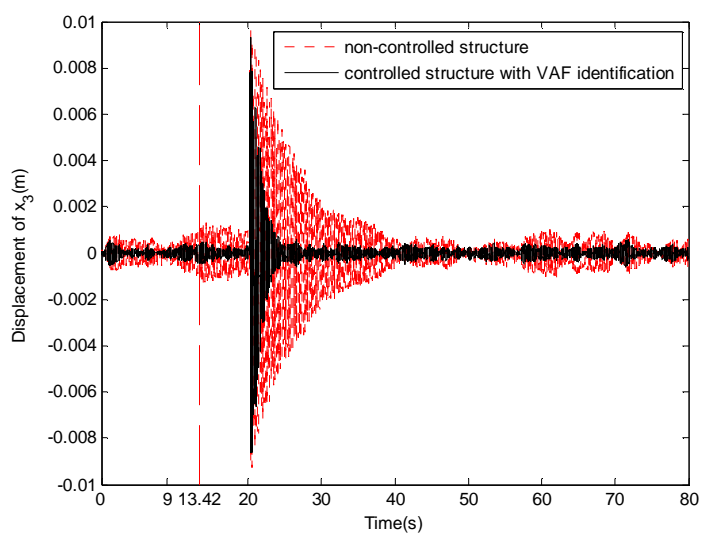

(a)

Figure 12 In the case of updated controller $\mathbf{G}$ and observer $\mathbf{L}$ with VAF identification, with disturbance: (a) displacement of $x_{3}$ 


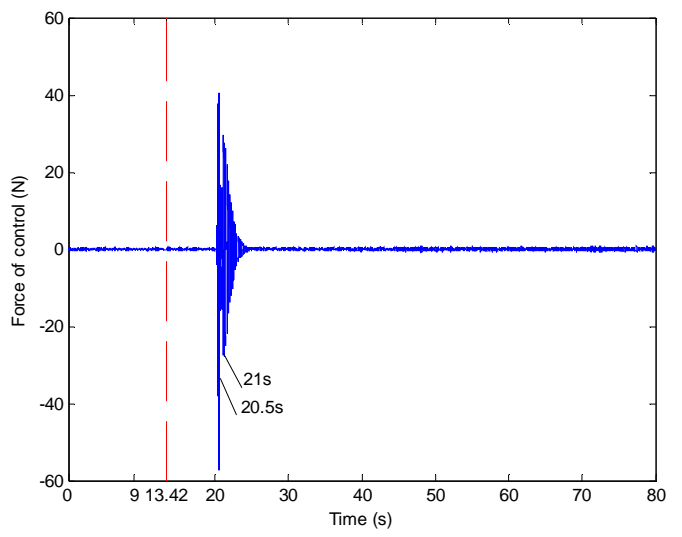

(b)

Figure 12 In the case of updated controller $\mathbf{G}$ and observer $\mathbf{L}$ with VAF identification, with disturbance: (b) force of control 
Table1: Physical and modal characteristics of the structure (initial state)

\begin{tabular}{cccc}
\hline $\begin{array}{c}\text { Mass of pendulums } \\
(\mathrm{Kg})\end{array}$ & $\begin{array}{c}\text { Stiffness of } \\
\text { spring }(\mathrm{N} / \mathrm{m})\end{array}$ & $\begin{array}{c}\text { Length of stems } \\
(\mathrm{m})\end{array}$ & $\begin{array}{c}\text { Mass of stems } \\
(\mathrm{Kg})\end{array}$ \\
$\mathrm{m}_{1}=2.61$ & $\mathrm{k}_{1}=13182$ & $\mathrm{Lt}_{1}=0.414$ & $\mathrm{Mt}_{1}=0.305$ \\
$\mathrm{~m}_{2}=2.61$ & $\mathrm{k}_{2}=13182$ & $\mathrm{Lt}_{2}=0.414$ & $\mathrm{Mt}_{2}=0.305$ \\
$\mathrm{~m}_{3}=0.875$ & $\mathrm{k}_{3}=13182$ & $\mathrm{Lt}_{3}=0.431$ & $\mathrm{Mt}_{3}=1.205$ \\
\hline Modal damping ratios & \multicolumn{2}{c}{ Initial eigen frequencies } \\
$\xi_{1}=2.6 .10^{-3}$ & \multicolumn{2}{c}{$\mathrm{f}^{\text {rd }}$ pendulum mass at the bottom location $)$} \\
$\xi_{2}=2.6 .10^{-3}$ & \multicolumn{2}{c}{$\mathrm{f}_{2}=17.5 \mathrm{~Hz}$} \\
$\xi_{3}=2.6 .10^{-3}$ & $\mathrm{f}_{3}=23.9 \mathrm{~Hz}$ \\
\hline
\end{tabular}

